\title{
Time-resolved in-situ investigations of reactive sputtering processes by grazing incidence X-ray absorption spectroscopy
}

\section{Dirk Lützenkirchen-Hecht* and Ronald Frahm}

Fachbereich C - Experimentalphysik, Bergische Universität Wuppertal, Gaußstr. 20, 42097 Wuppertal, Germany

*Corresponding author: e-mail: dirklh@uni-wuppertal.de

Phone: +49 (0)202 4393103

Fax: +49 (0)202 4393101

\section{Abstract:}

We have applied the time-resolved grazing incidence X-ray absorption fine structure technique to study in-situ the atomic short range order and the electronic structure of reactively sputter deposited thin films. Results obtained during the reactive deposition of amorphous Ta-pentoxide thin films deposited in oxygen containing atmospheres will be presented. A new calculation scheme for a detailed reflection mode EXAFS data analysis giving bond distances, coordination numbers and Debye-Waller factors is presented. The atomic short range structure of the amorphous $\mathrm{Ta}_{2} \mathrm{O}_{5}$ thin films is compared to that of crystalline $\beta-\mathrm{Ta}_{2} \mathrm{O}_{5}$.

Keywords: X-ray absorption spectroscopy, sputter deposition, thin films, in-situ analysis 


\section{Introduction:}

Thin film preparation by sputtering comprises the bombardment of a target with accelerated ions, the relief of target material into the gas phase and its deposition on an eventually heated or cooled substrate. Compound materials such as oxides or nitrides may be deposited if a reactive species such as oxygen or nitrogen is present in the gas atmosphere. The volume and surface microstructure of reactively sputtered thin films is known to depend strongly on all the details of the deposition process such as e.g. the substrate material and its temperature, the gas pressure and the deposition rate $[1-6]$. In parallel to the observed structural changes, many other physico-chemical properties such as the transition temperature for superconducting films [7], the hardness [8], magnetic [5] or optical properties [9] may vary as a function of the deposition conditions. Thus in-situ techniques are highly desirable for the study and the understanding of the growth phenomena during reactive sputtering. In contrast to electron-based techniques which generally require ultrahigh vacuum conditions, photon based techniques such as X-ray diffraction and X-ray absorption spectroscopy are compatible to the process conditions during sputtering, i.e. a rather high vacuum pressure of typically some $\mathrm{Pa}$ and the presence of ions and electrons in the sputter plasma.

In this study, time resolved grazing incidence X-ray absorption spectroscopy was used to study the growth of tantalum pentoxide thin films by reactive magnetron sputtering in $\mathrm{Ar}-\mathrm{O}_{2}$ mixtures. Ta-oxide has many physical properties that are attractive for materials science, especially for applications in microelectronic devices. For example, metal-oxidesemiconductor devices with extremely low leakage currents and high breakdown strengths can be realized using thin insulating $\mathrm{Ta}_{2} \mathrm{O}_{5}$ films instead of using oxidized silicon $[10,11]$. In addition, $\mathrm{Ta}_{2} \mathrm{O}_{5}$ has a high refractive index [12], a large band-gap $\mathrm{E}_{\mathrm{g}} \approx 4.2 \mathrm{eV}$. and is almost absorption free in the wavelength range from $300 \mathrm{~nm}$ to $2.0 \mu \mathrm{m}$ [13], allowing e.g. its use as antireflective coating for eyeglasses, solar cells and charge-coupled devices [14]. 
Furthermore, $\mathrm{Ta}_{2} \mathrm{O}_{5}$ can be useful as corrosion resistant protective coating [15], as piezoelectric material [16] and in thin film sensors [14].

Many investigations have been performed in the past to analyze the structure of the $\mathrm{Ta}_{2} \mathrm{O}_{5}$ thin films as a function of the preparation conditions since it is reasonable that the their physicochemical properties are strongly dependent on the microstructure of the film from a length scale of the crystallographic unit cell (i.e. some $\AA$ ) to the order of the film thickness. We have therefore conducted the first in-situ experiments during the reactive sputter deposition of Taoxide on float glass substrates. Especially the initial stages of growth were analysed.

\section{Experimental details:}

Ta-oxide thin films were deposited on smooth float glass substrates (5-6 $\AA$ surface roughness [17]) in pure $\mathrm{O}_{2}$ and $\mathrm{Ar} / \mathrm{O}_{2}$ atmospheres $(\mathrm{p} \approx 1.2 \mathrm{~Pa})$ using a Ta metal target and a magnetron source of $55 \mathrm{~mm}$ diameter with a power of up to $30 \mathrm{~W}$. The reactive sputter deposition took place in a small vacuum chamber that was optimized for in situ investigations of sputtered thin films with X-rays [18]. While the top flange of this chamber accommodates the magnetron sputter source with the Ta-target, the base of the chamber has flanges for the sputter gas inlet, a connection to a turbo-molecular pump and electrical feedthroughs e.g. for the measurement of the substrate temperature. Two large area Kapton foils served as windows for the incoming and the reflected X-ray beam. The base pressure of the vacuum vessel was well below $10^{-4} \mathrm{~Pa}$. More details of the cell are given elsewhere [18].

Ex-situ X-ray photoelectron spectroscopy (XPS) was performed after the removal of the films from the sputter chamber and a transfer to a surface analytical system. The experiments gave a $\mathrm{Ta} / \mathrm{O}$ ratio of about $(0.39 \pm 0.01) / 1$ independent from the gas composition during the sputter deposition. This indicates that stoichiometric $\mathrm{Ta}_{2} \mathrm{O}_{5}$ was formed. These XPS-investigations also prove the presence of a minor surface contamination with carbon species. Additional X- 
ray diffraction experiments showed that the prepared $\mathrm{Ta}_{2} \mathrm{O}_{5}$ thin films are generally $\mathrm{X}$-ray amorphous.

The X-ray absorption experiments were performed at the bending magnet station RÖMO II [19] at the DORIS III storage ring at HASYLAB (Hamburg, Germany) operating with about 100-150 $\mathrm{mA}$ of stored $4.45 \mathrm{GeV}$ positrons. A double-crystal monochromator equipped with two flat Si(111) crystals was used. Surface sensitive reflection mode EXAFS data were collected at room temperature in the vicinity of the $\mathrm{Ta}_{3}$-edge $(9881.2 \mathrm{eV})$. Incident and reflected intensities were measured by means of $\mathrm{N}_{2}$ and Ar filled ionization chambers. The incoming beam was collimated vertically by a slit system to about $120-250 \mu \mathrm{m}$, and a second slit was placed in front of the second ionization chamber to reduce the amount of non-specular scattered X-rays. A beam stop was installed to shield the second detector from the direct beam. The measured intensity ratios between the second and the first ionization chamber were routinely normalized with respect to the corresponding signals without sample in order to obtain absolute reflectivities. Additional geometric corrections were applied for small grazing angles, for which only a part of the monochromatic beam impinges on the sample (see e.g. [20]). A time resolution of about 20-30 s for scans consisting of an energy range of about $500-600 \mathrm{eV}$ with ca. 800-1000 data points was obtained by using the quick-scanning EXAFS mode, in which the monochromator is moved continuously from smaller to higher photon energies and the spectrum is measured on the fly [19]. A Ta metal foil was measured in transmission between the second and a third ionization chamber in order to calibrate the energy scale of the monochromator simultaneously with each sample spectrum. Reference spectra were obtained in transmission mode from a Ta metal foil and crystalline $\beta-\mathrm{Ta}_{2} \mathrm{O}_{5}$ powders. In the present study, fluorescence detection - which is in principle possible using the sputter cell (see [18]) - was not considered, but will be applied in forthcoming investigations under suitable circumstances. Due to the relatively low photon flux of the bending magnet beamline used in the current study, the limited solid angle 
usable by a fluorescence detector and the small data collection per data point (which is on the hand necessary for our time resolved studies) the fluorescence signal is not sufficient for a meaningful EXAFS data analysis [21].

\section{Results and discussion:}

Raw reflection mode EXAFS data measured in-situ during the reactive deposition of $\mathrm{Ta}_{2} \mathrm{O}_{5}$ in an $\mathrm{Ar} / \mathrm{O}_{2}$-mixture are presented in Fig. 1. The time resolution was about $25 \mathrm{~s}$ for each scan. The grazing angle was set to $\phi=0.223^{\circ}$ which is slightly above the critical angle $\phi_{\mathrm{c}}$ of total reflection of the float glass substrate $\left(\phi_{C}(\right.$ Glass $\left.) \approx 0.18^{\circ}\right)$, but below that of $\mathrm{Ta}_{2} \mathrm{O}_{5}\left(\phi_{\mathrm{C}}\left(\mathrm{Ta}_{2} \mathrm{O}_{5}\right) \approx 0.28^{\circ}\right)$, which ensures a high sensitivity of the experiment to the growing thin film. With an incidence angle smaller than $\phi_{c}$ of the substrate, the reflectivity would be high even for the uncoated glass, resulting in only small changes of the reflectivity when the Ta-oxide film is deposited. On the other hand, if the grazing angle is larger than $\phi_{c}$ of $\mathrm{Ta}_{2} \mathrm{O}_{5}$, the reflectivity remains small during the oxide layer deposition. Thus the choice of the incidence angle is a critical issue for the success of the experiment. As can be seen in Fig. 1, the reflectivity significantly increases in the course of the Ta-oxide layer deposition, and the increase of the edge step can easily be associated with the increasing film thickness.

From Fig. 2 it can be deduced that the experimental data quality is high enough to resolve the weak EXAFS oscillations belonging to the amorphous samples even for films of some few nm thickness only. Here, background subtracted data $\Delta \mathrm{R}=\mathrm{R}-\mathrm{R}_{0}$ are shown, where $\mathrm{R}_{0}$ is a smooth background, and the data were transformed from the energy scale to the photoelectron wave number scale $\mathrm{k}$ and weighted with $\mathrm{k}^{3}$ to stress the weak oscillations. According to the detailed analysis of specular reflectivity measurements ( $\phi-2 \phi$-scans) with fixed photon energy, the deposition rate is about $0.018 \mathrm{~nm} / \mathrm{s}$, i.e. the two shown spectra correspond to thicknesses of about 
$1.2 \mathrm{~nm}$ and $1.8 \mathrm{~nm}$, respectively. It is worth mentioning that a pronounced fine structure can be measured even for these thin films consisting of some few monolayers only.

These fine structures were subsequently subjected to a Fourier-transform (FT) and typical results are presented in Fig. 3. As can be seen, the intensities of characteristic features e.g. at ca. $1.5 \AA$ and $3.0 \AA$ radial distance increase as a function of the sputter time. This can be seen in more detail in the insert of Fig. 3, where the intensity of the first peak - which belongs to the first TaO-coordination of the oxide film - is plotted as a function of the sputtering time. While standardized data processing routines are available for the evaluation of transmission mode EXAFS data (see e.g. [21]), the situation is generally more complicated for reflection mode experiments because both the real $(\delta(E))$ and the imaginary part $(\beta(E))$ of the complex refractive index

$$
n(E)=1-\delta(E)-i^{*} \beta(E)
$$

contribute to the reflectivity. Thus, the reflectivity strongly depends on the grazing angle and the film thickness, and a simple normalization of the reflection mode EXAFS data is impossible (see e.g. [22]) - this is the reason why we only considered $\Delta R(E)$ data and their Fourier-transforms. Furthermore, the absorption coefficient $\mu$

$$
\mu=4 \pi \beta / \lambda
$$

and its fine structure has to be extracted from reflectivity EXAFS data, which is possible only for thin films of sufficient thickness (see e.g. [22-25]). However, this is not feasible for thin films with a thickness smaller than the penetration depth of the X-rays. In this case, the X-ray reflectivity has to be modelled using the Fresnel-theory for layered systems. In the past, the calculations have used refractive indices $\mathrm{n}(\mathrm{E})$ (eq. (1)) which were extracted from experimental data of reference compounds (e.g. [26]), implying that spectra of amorphous thin film samples cannot be modelled properly by such simple calculations. Thus, we calculated $n(E)$ data on a pure ab-initio basis as follows below. This approach differs significantly from procedures 
reported in the literature (e.g. [22]) because the absorption is not extracted from the reflectivity data, and the fit is performed using the reflectivity and not the absorption data.

Assuming that the short range order structure around the absorbing Ta-atom is characterized by the structural parameters of the first coordination shell $\mathrm{R}_{1}, \mathrm{~N}_{1}$ and $\sigma_{1}$, denoting the interatomic distance, coordination number and Debye-Waller term, respectively, we determine the extended X-ray absorption fine structure $\chi(\mathrm{k})$ by an ab-initio calculation using FEFF [27]. After transformation to the energy scale (using $\mathrm{E}_{0}=9881.2 \mathrm{eV}$ ), this $\chi(\mathrm{E})$-spectrum was converted to $\Delta \beta(E)$ using eq. (2) and superimposed on a structureless, atomic background, the calculation of which was done by using values for $\delta_{0}(E)$ and $\beta_{0}(E)$ in the Cromer-Liberman (CL) approximation [28], i.e. $\beta(E)=\beta_{0}(E)+\Delta \beta(E)$. The CL-data were convoluted with a Lorentzian of tabulated linewidth (4.88 eV in our case, see [29]) in order to account for core-hole lifetime effects as described in more detail in [30]. This calculated Xray absorption spectrum was subsequently Kramers-Kronig-transformed using the computer code described in [30], giving finally the complex index of refraction associated with the thin film material. It has to be stressed here that the Kramers-Kronig transform is only necessary for the calculation of the refractive index of the thin film. Due to the fact that a difference Kramers-Kronig transform is applied which uses the CL-data for the atomic part of both $\delta_{0}(\mathrm{E})$ and $\beta_{0}(E)$, the values obtained are expected to quite accurate. In a last step, reflection mode EXAFS spectra were calculated applying the Fresnel theory for the glancing angles $\phi$ defined by the experiment with a high precision of about $\pm 0.001^{\circ}$ and making use of the so calculated index of refraction of the film and Cromer-Lieberman data for the glass substrates. Because of the smooth surfaces of the sputter deposited $\mathrm{Ta}_{2} \mathrm{O}_{5}$-films (roughness $<7 \AA$ ), the influence of the surfaces on the thin film reflectivity only has a small influence on the reflectivity EXAFS as we have shown in a previous study [31]. Thus it was neglected in the present study. 
This way, it is possible to fit the reflection mode EXAFS data measured in-situ during the reactive sputter deposition of $\mathrm{Ta}_{2} \mathrm{O}_{5}$ thin films by adjusting the film thickness $\mathrm{d}, \mathrm{R}_{1}, \mathrm{~N}_{1}$ and $\sigma_{1}$ until a best fit is obtained. Typical fit results for some selected deposition times are depicted in Fig. 4 in R-space representation. For all these calculations, $\mathrm{R}_{1}=1.939 \AA, \mathrm{N}_{1}=4.65$, and $\sigma_{1}=0.1 \AA$ were used and only the film thickness has to be varied for a proper fit of the experimental data. It should be mentioned here that the values for $\mathrm{R}_{1}, \mathrm{~N}_{1}$ and $\sigma_{1}$ agree quantitatively with previous ex-situ experiments [32]. For comparison, the coordination number of the polycrystalline reference compound $\beta-\mathrm{Ta}_{2} \mathrm{O}_{5}$ is $\mathrm{N}_{1}=6$ as expected, and $\mathrm{R}_{1} \approx 1.97 \AA$ as well as $\sigma_{1} \approx 0.1 \AA$ are reasonable values for this crystalline material. In contrast, two recent EXAFS studies dealing with $\mathrm{Ta}_{2} \mathrm{O}_{5}$ thin films have determined a significantly larger Ta-O bond distance of about $2.05 \AA[33,34]$. However, the quality of our experimental data exhibits less noise than these studies. In addition, only a small k-range could be used for the data fitting in one of the mentioned studies [33], and the coordination numbers derived in that paper $\left(\mathrm{N}_{1} \approx 6.1-7.8\right)$ seem to be too large, while the value for the mean square displacement $\sigma_{1}$ is similar to those determined here. The interatomic distance determined in the second study [34] is close to that calculated from the spectra presented here, while the value for $\sigma_{1} \approx 0.1 \AA$ is identical. Thus we can conclude that the values determined here are well founded.

In general, the thin films reveal smaller bond distances compared to the bulk reference; this is a typical feature for amorphous samples (see e.g. $[35,36]$ ). In addition, a significantly reduced coordination number $\mathrm{N}_{1}$ and a slightly reduced $\sigma_{1}$ were determined for the thin film samples. This apparently reduced coordination number together with the smaller bond distance is a further indication for a highly disordered material and originates from the highly asymmetric pair distribution functions in amorphous solids: On the one hand, the repulsive part of the pair potential gives narrower distance distributions (i.e. smaller values for $\sigma$ ). On the other hand, EXAFS probes high values in momentum space and is therefore very sensitive to a broadening 
of the pair correlation functions $[35,36]$. Due to the fact that all the films prepared in this study can be described by an identical set of parameters, it can be concluded that the atomic short range order of the growing $\mathrm{Ta}_{2} \mathrm{O}_{5}$ thin films is not changing as a function of thickness. This is further supported by the fact that the growth rate, i.e. the slope of the determined thickness as a function of the deposition time is almost constant for the whole experiment - in agreement with the constant sputtering current during the deposition - and the determined value $(1.73 \pm 0.03) * 10^{-2} \mathrm{~nm} / \mathrm{s}$ corresponds well to the data mentioned above. In addition, the reproducibility of room temperature experiments is excellent and agrees quantitatively with results obtained using independent other methods.

From a closer inspection of the FT-data presented in Fig. 3 it is, however, clear that the determined short range order structural parameters exhibit certain errors, as can be expected e.g. from the scatter in the radial distance of the nearest neighbour peak which amounts to about $\pm 0.02 \AA$. In a similar way, the deviation of the derived thickness values from the linear slope as a function of time also indicates that the nearest neighbour number is influenced: Both the thickness as well as $\mathrm{N}_{1}$ affect the amplitude of the nearest neighbour peak in the FT of the simulated data in a very similar way, and thus the film thickness for the simulation should be taken from an independent measurement using e.g. a quartz thickness monitor and $\mathrm{N}_{1}$ should be varied until a good fit is obtained.

\section{Conclusions:}

Amorphous $\mathrm{Ta}_{2} \mathrm{O}_{5}$-thin films were prepared on float glass substrates by reactive magnetron sputtering in $\mathrm{O}_{2} / \mathrm{Ar}$ mixtures. The structure of the films was investigated in-situ using time resolved surface sensitive reflection mode X-ray absorption spectroscopy. The experimental data were modelled by using a new fit procedure which indicates that the atomic short range order structure of the films is independent from the film thickness. The nearest neighbour Ta-

O bond distance amounts to ca. $1.94 \AA$, and a coordination number $\mathrm{N}_{1}=4.65$ and $\sigma_{1}=0.1 \AA$ 
were determined even for films of less than $1 \mathrm{~nm}$ thickness. These values obtained in-situ agree quantitatively with previous ex-situ experiments.

\section{Acknowledgements:}

We would like to thank K. Bruder, U. Haake and P. Keil for their help at the beamline. The experiments were financially supported by HASYLAB and the MWF Nordrhein-Westfalen. 


\section{$\underline{\text { References: }}$}

[1] J.A. Thornton. Vac. Sci. Technol. 11 (1974) 666. Ann. Rev. Mater. Sci. 7 (1977) 239.

[2] M. Pakala, R.Y. Lin. Surf. Coat. Technol. 81 (1996) 233.

[3] K.H. Yoon, J.-W. Choi, D.-H. Lee. Thin Solid Films 302 (1997) 116.

[4] I. Hotovy, J. Huran, J. Janik, A.P. Kobzev. Vacuum 51 (1998) 157.

[5] B.M. Barnes, J.J. Kelly, J.F. MacKay, W.L. O`Brian, M.G. Lagally. IEEE Trans. Magn. 36 (2000) 2948.

[6] P.R. Willmott. Prog. Surf. Sci. 76 (2004) 163.

[7] S.A. Wolf, I.L. Singer, E.J. Cukauskas, T.L. Francaville, E.F. Skelton. J. Vac. Sci. Technol. 17 (1980) 411.

[8] K.S. Havey, J.S. Zabinski, S.D. Walck. Thin Solid Films 303 (1997) 238.

[9] S.G. Yoon, H.K. Kim, M.J. Kim, H.M. Lee, D.H. Yoon. Thin Sol. Films 475 (2005) 239.

[10] R.A.B. Devine, L. Vallier, J.L. Autran, P. Paillet, J.L. Leray. Appl. Phys. Lett. 68 (1996) 1775.

[11] G.B. Alers, R.M. Fleming, Y.H. Wong, B. Dennis, A. Pinczuk, G. Redinba, R. Urdahl, E. Ong, Z. Hasan. Appl. Phys. Lett. 72 (1998) 1308.

[12] S. Boughaba, G.I. Sproule, J.P. McCaffrey, M. Islam, M.J. Graham. Thin Solid Films 358 (2000) 104.

[13] J.D. Taylor-Kruschwitz, W.T. Pawlewicz. Appl. Optics 36 (1997) 2157.

[14] C. Chaneliere, J.L. Autran, R.A.B. Devine, B. Balland. Mater. Sci. Eng. R22 (1998) 269.

[15] P.M. Natishan, E. McCafferty, P.R. Puckett, S. Michel. Corros. Sci. 38 (1996) 1043.

[16] Y. Nakagawa, Y. Gomi. Appl. Phys. Lett. 46 (1985) 139.

[17] P. Keil, D. Lützenkirchen-Hecht, R. Frahm. HASYLAB annual report (2000) 495. 
[18] D. Lützenkirchen-Hecht, K. Bruder, U. Haake, P. Keil, C. Markert, C. Ringpfeil, R. Frahm. Rev. Sci. Instrum. 76 (2005) 073905.

[19] R. Frahm. Rev. Sci. Instr. 60 (1989) 2515.

[20] A. van der Lee. Solid State Sciences 2 (2000) 257.

[21] D.C. Koningsberger and R. Prins, X-ray absorption: Principles, Applications, Techniques of EXAFS, SEXAFS and XANES, Wiley, New York, 1988.

[22] S.M. Heald, H. Chen and J.M. Tranquada. Phys. Rev. B 38 (1988) 1016.

[23] S. Pizzini, K. J. Roberts, G. N. Greaves, N. Harris, P. Moore, E. Pantos, R. J. Oldman. Rev. Sci. Instrum. 60 (1989) 2525.

[24] B. Poumellec, R. Cortes, F. Lagnel, G. Tourillon. Physica B 158 (1989) 282.

[25] P. Borthen, H.-H. Strehblow. J. Phys.: Cond. Mat. 7 (1995) 3779.

[26] P. Borthen, H.-H. Strehblow. J. Phys. IV (France) 7 (1997) C2-695.

[27] J.J. Rehr, R.C. Albers, S.I. Zabinski, Phys. Rev. Lett. 69 (1992) 3397.

[28] D.T. Cromer, D. Libermann. J. Chem. Phys. 53 (1970) 1891.

[29] M.O. Krause, J.H. Oliver. J. Phys. Chem. Ref. Data 2 (1979) 329.

[30] J. O. Cross, M. Newville, J.J. Rehr, L.B. Sorensen, C.E. Bouldin, G. Watson, T. Gouder, G.H. Lander, M.I. Bell. Phys. Rev. B 58 (1998) 11215.

[31] P. Keil, D. Lützenkirchen-Hecht, R. Frahm. Phys. Scr. T115 (2005) 246.

[32] D. Lützenkirchen-Hecht and R. Frahm. J. Synchrotron Rad. 8 (2001) 478.

[33] H. Kimura, J. Mizuki, S. Kamiyama, H. Suzuki. Appl. Phys. Lett. 66 (1995) 2209.

[34] A. Kuzmin, R. Kalendarev, J. Purans. Proc. SPIE 5122 (2003) 80.

[35] G.S. Cargill, W. Weber, R.F. Boehme, R.Frahm. in EXAFS and Near Edge Structure, A. Bianconi, L. Incoccia and S. Stipcich (Eds.), Springer Verlag, Berlin (1983) 277.

[36] R. Frahm, R. Haensel, P. Rabe. J. Phys. F: Met. Phys. 14 (1984) 1333. 


\section{Figure captions:}

Fig. 1:

Raw in-situ reflection mode $\left(\phi=0.223^{\circ}\right) \mathrm{Ta}_{3}$-edge spectra of the amorphous Ta-oxide layers during reactive sputter deposition at room temperature. Each spectrum was measured in about 25 s using the Quick-scanning EXAFS technique. No data processing or reduction such as smoothing or averaging was applied to the experimental data points.

Fig. 2:

$\mathrm{k}^{3}$-weighted reflection mode fine structure of two single spectra. The dotted and the full line correspond to sputtering times of about $67 \mathrm{~s}$ and $100 \mathrm{~s}$, respectively. The peaked structure at about $3 \AA^{-1}$ correspond to a glitch of the monochromator. The film thickness amounts to ca. $1.2 \mathrm{~nm}$ and $1.8 \mathrm{~nm}$, respectively.

Fig. 3:

Evolution of the magnitude of the Fourier-transforms of the fine structure oscillations extracted from the reflection mode spectra presented in Fig. 1 as a function of the sputter time. In the insert, the growth of the peak at about $1.5 \AA$ radial distance $\left(\mathrm{R}_{1}\right)$ belonging to the first Ta-O coordination of the growing oxide film is shown. $60 \mathrm{~s}$ sputter time correspond to about $1.08 \mathrm{~nm}$ film thickness. The data are not phase shift corrected. The k-range for the Fourier transformation is $1.7 \AA^{-1} \leq \mathrm{k} \leq 9 \AA^{-1}$.

Fig. 4:

Examples for the fitting of the experimental reflection mode EXAFS data for different growth stages (deposition times) for a deposition at room temperature. Fit data were calculated on an ab-initio basis assuming $\mathrm{R}_{1}=1.939 \AA, \mathrm{N}_{1}=4.65, \sigma_{1}=0.1 \AA$. 